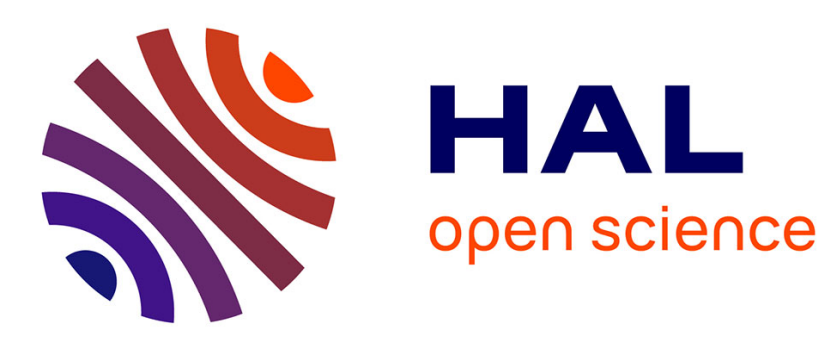

\title{
Tomographie de diffraction et synthèse de Fourier à maximum d'entropie
}

A. Mohammad-Djafari, G. Demoment

\section{To cite this version:}

A. Mohammad-Djafari, G. Demoment. Tomographie de diffraction et synthèse de Fourier à maximum d'entropie. Revue de Physique Appliquée, 1987, 22 (2), pp.153-167. 10.1051/rphysap:01987002202015300 . jpa-00245527

\section{HAL Id: jpa-00245527 https://hal.science/jpa-00245527}

Submitted on 1 Jan 1987

HAL is a multi-disciplinary open access archive for the deposit and dissemination of scientific research documents, whether they are published or not. The documents may come from teaching and research institutions in France or abroad, or from public or private research centers.
L'archive ouverte pluridisciplinaire HAL, est destinée au dépôt et à la diffusion de documents scientifiques de niveau recherche, publiés ou non, émanant des établissements d'enseignement et de recherche français ou étrangers, des laboratoires publics ou privés. 


\title{
Tomographie de diffraction et synthèse de Fourier à maximum d'entropie
}

\author{
A. Mohammad-Djafari et G. Demoment \\ Laboratoire des Signaux et Systèmes (CNRS-ESE-UPS), Plateau du Moulon, 91190 Gif-sur-Yvette, France
}

(Reçu le 28 avril 1986, révisé le 16 juillet, accepté le 16 octobre 1986)

\begin{abstract}
Résumé. - En tomographie de diffraction, le théorème de Radon généralisé permet de relier la Transformée de Fourier (TF) du champ diffracté mesuré sur une ligne droite à la TF 2-D de l'objet sur un contour algébrique, qui est un demi-cercle dans le cas des approximations de Born ou de Rytov. Pour améliorer la résolution insuffisante des méthodes d'interpolation usuellement utilisées, nous proposons une méthode de synthèse de Fourier par Maximum d'Entropie (ME). Pour cela on définit l'entropie d'une image considérée comme une fonction de $\mathbb{R}^{2}$ dans $\mathbb{C}$. Une statistique du $\chi^{2}$ est ajoutée à l'entropie pour la prise en compte du bruit. Un algorithme de reconstruction utilisant les techniques du gradient conjugué est présenté et, finalement, une comparaison est faite entre des résultats obtenus par les méthodes classiques de reconstruction et notre méthode.
\end{abstract}

\begin{abstract}
In diffraction tomography, the generalized Radon theorem relates the Fourier Transform (FT) of the diffracted field to the two-dimensional FT of the diffracting object. The relation stands on algebraic contours, which are semi-circles in the case of Born or Rytov first order linear approximations. To increase the insufficient resolution of generally used interpolation methods we propose a Maximum Entropy Fourier synthesis method. To do this, we give a new definition for the entropy of an object considered as a function of $\mathbb{R}^{2}$ to $\mathbb{C}$. To take into account the presence of noise, a $\chi^{2}$ statistics is added to the entropy measure. The objective function thus obtained is minimized using variational techniques and a conjugate-gradient iterative method. Some simulated results are given which compare this new method with the classical ones.
\end{abstract}

\section{Introduction.}

La tomographie à onde diffractée (micro-onde ou ultrasonore) est une technique qui se développe rapidement dans le domaine de l'imagerie médicale et industrielle.

La généralisation du théorème de Radon, utilisé en tomographie à rayons $\mathrm{X}$, à la tomographie à onde diffractée permet de connaître comment on remplit l'espace de Fourier de l'objet diffractant à partir de mesures du champ qu'il diffracte. On peut alors en principe reconstruire l'objet par une transformation de Fourier (TF) inverse.

Il faut bien voir cependant que la tomographie de diffraction soulève deux sortes de problèmes dont la nature est différente. La propagation des ondes dans les différents milieux du problème est en effet décrite par des équations différentielles dont on ne possède pas, en général, de solution explicite reliant le champ diffracté à l'objet.

Une première étape consiste donc à faire un développement limité (le plus souvent actuellement au premier ordre) de la solution en introduisant des hypothèses

REVUE DE PHYSIQUE APPLIQUÉE. - T. 22, N`2, FÉVRIER 1987 supplémentaires sur le problème (objet peu diffractant, variations d'indice faibles, etc.). Cette étape a pour conséquence d'introduire une relation explicite entre les données mesurées (champ diffracté ou «projections ») et l'objet à reconstruire, faisant souvent apparaître la TF de l'objet (théorème de Radon). Mais le nombre nécessairement limité des projections fait que l'on a qu'une information incomplète sur l'objet.

La deuxième étape consiste alors à développer une méthode d'extrapolation et d'interpolation permettant d'obtenir une solution unique, et si possible stable, du problème de reconstruction. Ceci ne peut se faire qu'en introduisant, explicitement ou implicitement, une information a priori sur l'objet à reconstruire.

Dans cet article, nous rappelons dans une première partie les principales méthodes d'approximation linéaire utilisées, ainsi que le théorème de Radon généralisé. Dans une deuxième partie nous analysons les méthodes de reconstruction tomographiques existantes afin de mettre en évidence leurs hypothèses implicites et les limitations qu'elles entraînent. Enfin, dans une troisième partie, nous proposons une nouvelle 
méthode de reconstruction basée sur le principe du maximum d'entropie.

Un certain nombre de résultats de simulation sont donnés pour comparer les résultats de la reconstruction par les différentes méthodes et illustrer le comportement de notre méthode dans des situations défavorables.

\section{Principe de l'imagerie à onde diffractée.}

1.1 EQuations DE PROPAGATION ET APPROXIMATIONS LINÉAIRES. - Les modèles mathématiques pour la tomographie à onde diffractée ont été développés par Wolf [1] et Devaney [2] pour les ultrasons et par Bojarsky [3] et Richmond [4] pour les micro-ondes.

Dans un milieu homogène, les ondes ultrasonores ou électromagnétiques satisfont l'équation de Helmoltz

$$
\left[\nabla^{2}+k_{0}^{2}\right] u_{0}(\mathbf{r})=0
$$

où $k_{0}$ est la fréquence spatiale de l'onde plane et $u_{0}$ est le champ. En imagerie on s'intéresse à un milieu non homogène. Si on néglige les effets de la polarisation, la forme générale de l'équation d'onde devient

$$
\left[\nabla^{2}+k^{2}(\mathbf{r})\right] u(\mathbf{r})=0
$$

où $k(\mathbf{r})$, indice de réfraction du milieu, est défini par

$$
k^{2}(\mathbf{r})=k_{0}^{2}[1+f(\mathbf{r})]=\omega^{2} \mu(\mathbf{r}) \varepsilon(\mathbf{r})
$$

où $k_{0}$ est la valeur moyenne de la constante de propagation.

Pour résoudre l'équation (1) nous effectuons un développement au $1^{\text {er }}$ ordre. Pour cela nous définissons les changements de variables suivants

$$
v=g\left(u / u_{0}\right)=g(a) \text { et } v_{1}=u_{0} g(a)
$$

d'où

$$
\left[\nabla^{2}+k_{0}^{2}\right] v_{1}(\mathbf{r})=-u_{0} k_{0}^{2} h(\mathbf{r})
$$

où

$$
h(\mathbf{r})=f(\mathbf{r}) a(\partial g / \partial a)-\left(1 / k_{0}^{2}\right)\left(\partial^{2} g / \partial a^{2}\right)(\nabla a)^{2} .
$$

Les deux approximations bien connues de Born et de Rytov correspondent à deux choix différents de $g(a)$. Si les termes du second ordre sont négligés, on peut résoudre cette équation (4) à l'aide de la fonction de Green $G\left(r, r^{\prime}\right)$, qui, dans un espace à deux dimensions, est donnée par

$$
G\left(\mathbf{r}, \mathbf{r}^{\prime}\right)=G\left(\mathbf{r}-\mathbf{r}^{\prime}\right)=(j / 4) H_{0}^{(1)}\left[k_{0}\left(\left|\mathbf{r}-\mathbf{r}^{\prime}\right|\right)\right]
$$

où $H_{0}^{(1)}$ est la fonction de Hankel d'ordre zéro. Ainsi la solution se met sous la forme d'une équation intégrale de convolution

$$
v_{1}(\mathbf{r})=\int G\left(\mathbf{r}-\mathbf{r}^{\prime}\right) u_{0}\left(\mathbf{r}^{\prime}\right) h\left(\mathbf{r}^{\prime}\right) \mathrm{d} \mathbf{r}^{\prime} .
$$

Dans cette équation et par la suite le domaine d'intégration est le volume de l'objet.

Dans le cas de l'approximation de Born on a

$$
g(a)=a-1, \quad v=\left(u-u_{0}\right) / u_{0}, \quad v_{1}=\left(u-u_{0}\right)
$$

et

$$
h(\mathbf{r})=\left[1+u_{\mathrm{S}}(\mathbf{r}) / u_{0}(\mathbf{r})\right] f(\mathbf{r})
$$

où $u_{\mathrm{S}}(\mathbf{r})=u(\mathbf{r})-u_{0}(\mathbf{r})$ est le champ diffracté. Par approximation de Born d'ordre un, on entend $u_{\mathrm{S}}(\mathbf{r}) \ll u_{0}(\mathbf{r})$, ce qui veut dire que $h(\mathbf{r})=f(\mathbf{r})$. En notant $u_{S}^{1}(\mathbf{r})$ le champ diffracté obtenu par cette approximation, on a

$$
u_{S}^{1}(\mathbf{r})=\int G\left(\mathbf{r}-\mathbf{r}^{\prime}\right) u_{0}\left(\mathbf{r}^{\prime}\right) f\left(\mathbf{r}^{\prime}\right) \mathrm{d} \mathbf{r}^{\prime} .
$$

Dans le cas de l'approximation de Rytov on a

$$
g(a)=\log (a), \quad v=\log \left(u / u_{0}\right)
$$

et

$$
h(\mathbf{r})=f(\mathbf{r})+\left(1 / k_{0}^{2}\right)|\nabla a(\mathbf{r}) / a(\mathbf{r})|^{2} .
$$

Cette approximation est valable sous des contraintes un peu moins sévères. Par approximation de Rytov d'ordre un on entend $\left|\nabla V_{\mathrm{S}}(\mathbf{r})\right|^{2} \ll k_{0}^{2} f(\mathbf{r})$ ce qui nous donne avec les mêmes notations

$$
\begin{aligned}
v_{\mathrm{S}}^{1}(\mathbf{r})=u_{\mathrm{S}}(\mathbf{r}) / u_{0} & =\left[1 / u_{0}(\mathbf{r})\right] \times \\
& \times \int G\left(\mathbf{r}-\mathbf{r}^{\prime}\right) u_{0}\left(\mathbf{r}^{\prime}\right) f\left(\mathbf{r}^{\prime}\right) \mathrm{d} \mathbf{r}^{\prime} .
\end{aligned}
$$

Il est important de noter qu'en dépit de la similitude formelle entre les relations (8) et (10), les deux approximations correspondantes sont bien différentes [5].

Dans le cas de l'imagerie micro-onde on suppose que l'objet est illuminé par une onde plane polarisée rectilignement selon l'axe $\mathrm{Oz}$. On peut alors écrire [6]

$$
E_{z}(x, y)=E_{z}^{\mathrm{i}}(x, y)+E_{z}^{\mathrm{d}}(x, y)
$$

où $E_{z}^{\mathrm{i}}(x, y)$ et $E_{z}^{\mathrm{d}}(x, y)$ sont respectivement les composantes sur l'axe $z$ du champ incident et du champ diffracté, et

$$
\begin{aligned}
& E_{z}^{\mathrm{d}}(x, y)=\int_{S} J_{S}\left(x^{\prime}, y^{\prime}\right) \times \\
& \quad \times H_{0}^{(2)}\left\{k_{0}\left[\left(x-x^{\prime}\right)^{2}+\left(y-y^{\prime}\right)^{2}\right]^{1 / 2}\right\} \mathrm{d} x^{\prime} \mathrm{d} y^{\prime}
\end{aligned}
$$

où $J_{S}\left(x^{\prime}, y^{\prime}\right)=\left[k^{2}\left(x^{\prime}, y^{\prime}\right)-k_{0}^{2}\right] E_{z}\left(x^{\prime}, y^{\prime}\right)$ est la distribution de la densité de courant induite à l'intérieur de l'objet et où $S$ désigne la surface délimitant l'objet.

En définissant

et

$$
u(x, y)=E_{z}^{\mathrm{d}}(x, y) / E_{z}^{\mathrm{i}}(x, y)
$$

$$
h\left(x^{\prime}, y^{\prime}\right)=J_{S}\left(x^{\prime}, y^{\prime}\right) / E_{z}^{i}\left(x^{\prime}, y^{\prime}\right)
$$


on a

$$
\begin{aligned}
u(x, y)=\left[1 / E_{z}^{\mathrm{i}}(x, y)\right] \times \\
\quad \times \int_{S} H_{0}^{(2)}\left\{k_{0}\left[\left(x-x^{\prime}\right)^{2}+\left(y-y^{\prime}\right)^{2}\right]^{1 / 2}\right\} \\
\quad \times h\left(x^{\prime}, y^{\prime}\right) E_{z}^{\mathrm{i}}\left(x^{\prime}, y^{\prime}\right) \mathrm{d} x^{\prime} \mathrm{d} y^{\prime}
\end{aligned}
$$

Les équations (6) pour les ultrasons et (13) pour les micro-ondes sont des équations exactes, mais dans lesquelles $h\left(\mathbf{r}^{\prime}\right)$ et $h\left(x^{\prime}, y^{\prime}\right)$ sont des grandeurs qui ne dépendent pas seulement de l'objet, mais aussi du champ total à l'intérieur de l'objet. Ces équations ne sont donc pas des équations explicites vis-à-vis de la fonction caractérisant l'objet, et nous avons alors l'alternative suivante :

- ou bien nous considérons ces équations exactes, mais alors les grandeurs impliquées ne sont pas interprétables directement et de plus la résolution n'est pas aisée.

- ou bien nous nous intéressons à des grandeurs physiquement interprétables (constante de propagation ou permittivité complexe), mais il faut alors approcher ces équations par un développement au $1^{\text {er }}$ ordre pour obtenir des équations explicites reliant les grandeurs aux données mesurées.

Ainsi, si on s'intéresse au paramètre $h\left(x^{\prime}, y^{\prime}\right)$ qui représente la distribution de la densité de courant induite à l'intérieur de l'objet diffractant, normalisée par rapport au champ incident, l'équation (13) est une équation exacte et de même forme que (10). D'autre part si $E_{z}^{\mathrm{d}}\left(x^{\prime}, y^{\prime}\right) \ll E_{z}^{\mathrm{i}}\left(x^{\prime}, y^{\prime}\right)$, c'est-à-dire dans le cas de l'approximation de Born d'ordre un, $h\left(x^{\prime}, y^{\prime}\right)=$ $k^{2}\left(x^{\prime}, y^{\prime}\right)-k_{0}^{2}$ représente la variation de la constante de propagation de l'objet par rapport à celle du milieu dans lequel l'objet est immergé.

1.2 THÉORÈME DE RADON GÉNÉRALISÉ. - Dans l'approximation du premier ordre de Born, l'équation (8) peut être considérée comme la convolution de la fonction de Green $G(\mathbf{r})$ avec le produit de l'objet $f(\mathbf{r})$ et du champ incident $u_{0}(\mathbf{r})$.

Passant dans le domaine de Fourier, l'équation intégrale (8) nous donne

$$
\hat{u}(\boldsymbol{\omega})=\hat{G}(\boldsymbol{\omega})\left[\hat{f}(\boldsymbol{\omega}) * \hat{u}_{0}(\boldsymbol{\omega})\right]
$$

où * représente l'opérateur de convolution et où ^ désigne une transformée de Fourier (Fig. 1).

$$
\hat{f}(\boldsymbol{\omega})=\int f(\mathbf{r}) \exp (-j \omega \cdot \mathbf{r}) \mathrm{d} \mathbf{r} .
$$

Dans le cas de l'illumination avec une onde plane on a

$$
\hat{u}_{0}(\omega)=2 \pi \delta\left(\omega-\mathbf{k}_{0}\right)
$$

et

$$
\hat{f}(\omega) * \hat{u}_{0}(\boldsymbol{\omega})=2 \pi \hat{f}\left(\boldsymbol{\omega}-\mathbf{k}_{0}\right) .
$$
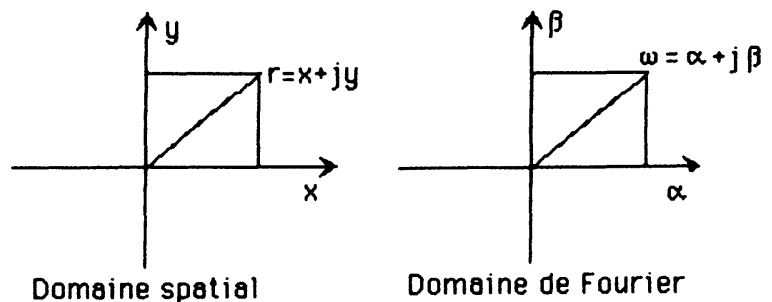

Fig. 1. - Coordonnées cartésiennes dans le domaine spatial et le domaine de Fourier.

[Cartesian coordinates in spatial and Fourier domain.]

L'effet de la convolution dans l'équation (14) est donc une multiplication dans le domaine de Fourier :

$$
\begin{aligned}
\hat{u}_{\mathrm{S}}(\boldsymbol{\omega})=\left[2 \pi /\left(\boldsymbol{\omega}^{2}-\right.\right. & \left.\left.\mathbf{k}_{0}^{2}\right)\right] \times \\
& \times \hat{f}\left(\boldsymbol{\omega}-\mathbf{k}_{0}\right) \exp \left(-j \boldsymbol{\omega} \cdot \mathbf{r}^{\prime}\right) .
\end{aligned}
$$

Limitons-nous, pour simplifier, au problème de l'imagerie 2-D, et considérons que le champ incident se propage en direction de l'axe $x$ positif, et que le champ diffracté est mesuré sur une ligne droite $x=x_{0}$. Ce champ est obtenu en calculant la TF inverse de l'équation (16) :

$$
\begin{aligned}
u_{S}(\mathbf{r})=(1 / 2 \pi) \int[\hat{f}(\boldsymbol{\omega} & \left.\left.-\mathbf{k}_{0}\right) /\left(\omega^{2}-\mathbf{k}_{0}^{2}\right)\right] \times \\
& \times \exp \left[j \omega \cdot\left(\mathbf{r}-\mathbf{r}^{\prime}\right)\right] \mathrm{d} \omega .
\end{aligned}
$$

Le calcul de cette intégrale peut être fait sur un contour autour des points singuliers $\omega^{2}-\mathbf{k}_{0}^{2}=\mathbf{0}$. En notant $\omega_{x}=\alpha$ et $\omega_{y}=\beta$, on obtient ainsi

$$
\begin{aligned}
u\left(x=x_{0}, y\right)=\int Q_{1}(\alpha) & \exp (j \alpha y) \mathrm{d} \alpha+ \\
& +\int Q_{2}(\alpha) \exp (j \alpha y) \mathrm{d} \alpha
\end{aligned}
$$

où

$$
\begin{gathered}
Q_{1,2}(\alpha)=[1 /( \pm j 2 \gamma)] \hat{f}(\alpha, \beta) \exp \left( \pm j \beta x_{0}\right), \\
\gamma=\left(k_{0}^{2}-\alpha^{2}\right)^{1 / 2} \text { et, } \beta=-k_{0}+\gamma
\end{gathered}
$$

En examinant ces deux équations on constate que $Q_{1}(\alpha)$ représente la solution en terme des ondes planes se propageant dans la direction des $x$ croissants, tandis que $Q_{2}(\alpha)$ représente la solution en terme des ondes planes se propageant dans la direction des $x$ décroissants. $Q(\alpha)$ est d'une part la TF des mesures $u_{S}\left(x_{0}, y\right)$ et d'autre part proportionnelle à la TF en 2-D de l'objet $f(\mathbf{r})$ évaluée sur le demi-cercle d'équation $\beta=-k_{0}+\left(k_{0}^{2}-\alpha^{2}\right)^{1 / 2}$. Dans le cas de la tomographie de transmission les mesures sont effectuées à $x=+x_{0}$, donc seule $Q_{1}(\alpha)$ intervient dans la solution (demi-cercle en trait plein dans la figure 2, tandis que dans la tomographie de réflexion seule $Q_{2}(\alpha)$ intervient dans la solution (demi-cercle en traits pointillés dans la Fig. 2). 


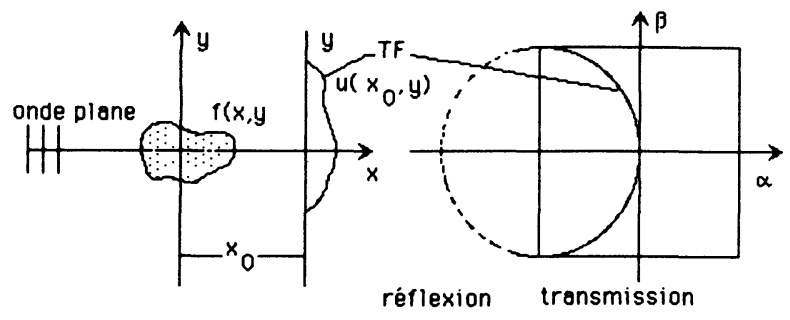

Fig. 2. - a) L'objet est illuminé par une onde plane et le champ diffracté est mesuré sur une ligne droite ; b) Relation entre la TF du champ diffracté et la TF 2-D de l'objet.

[a) The object is illuminated by a plane wave and the diffracted field is measured on a line perpendicular to the wave direction ; b) Relation between the FT of the diffracted field and the 2-D FT of the object.]

Ce résultat connu sous le nom de théorème de Radon généralisé relie ainsi la distribution de l'objet diffractant au champ diffracté mesuré sur une ligne droite.

Plusieurs procédures expérimentales peuvent être utilisées pour collecter les données et remplir le domaine de Fourier afin de reconstruire l'objet par une TF inverse. Ceci peut être fait soit en changeant la fréquence de l'onde incidente (rayon $k_{0}$ ) soit en tournant l'objet (l'ensemble des demi-cercles de rayons $k_{0}$ centrés sur un cercle de rayon $k_{0}$ ).

Par la suite nous supposerons que les données sont obtenues en tomographie de transmission et en tournant l'objet pour obtenir un nombre fini de "vues » repérées par des valeurs discrètes $\theta_{i}$ (Fig. 3). Ainsi l'équation générale de propagation s'écrit

$$
\left[\nabla^{2}+k_{0}^{2}\right] u^{\theta}(\mathbf{r})=-u_{0}(\mathbf{r}) h^{\theta}(\mathbf{r})
$$

et en se référant aux notations de la figure 3 on a

$$
\hat{h}\left(\theta\left(\begin{array}{l}
\beta \\
\alpha
\end{array}\right)\right)=(2 j \gamma) Q(\alpha) \exp \left(-j \beta x_{0}\right)
$$

où

$$
Q(\alpha)=\operatorname{TF}\left\{u_{S}\left(x_{0}, y\right)\right\}, \quad \gamma^{2}=\left(k_{0}^{2}-\alpha^{2}\right),
$$

$\beta=-k_{0}+\gamma$ et où $h(x, y)$ est une fonction 2-D proportionnelle à la distribution de l'objet.

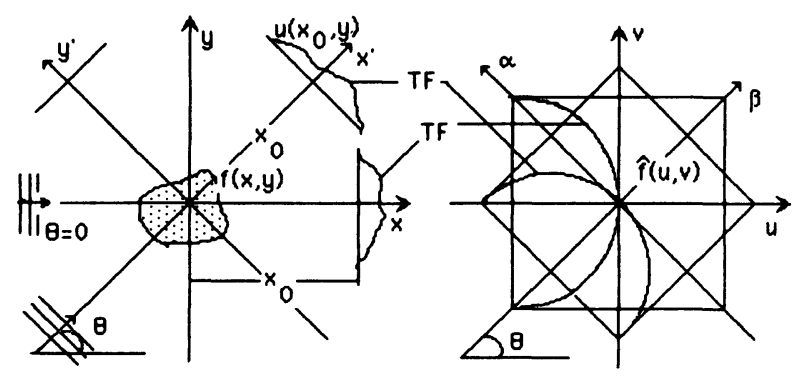

Fig. 3. - Relation entre la TF du champ diffracté et la TF 2-D de l'objet.

[Relation between the FT of the diffracted field and the 2-D FT of the object.]
Il existe deux approches différentes pour la résolution du problème de reconstruction.

i) Méthodes analytiques. - Dans ces méthodes, une solution du problème sous forme analytique est obtenue dans une première étape. Cette équation est ensuite discrétisée et résolue par des méthodes numériques. Dans le paragraphe 2.1 nous étudierons deux types d'algorithmes de ce genre qui ont été proposés dans la littérature.

ii) Méthodes algébriques. - Dans ces méthodes l'équation intégrale reliant l'objet aux mesures est discrétisée dès le départ, ensuite on résout le système d'équations linéaires ainsi obtenu. La méthode que nous proposons peut être classée dans cette famille.

\section{Méthodes de synthèse de Fourier.}

2.1 PrinCIPES DES MÉThOdES EXISTANTES. - Dans ce chapitre nous n'essayons pas de résoudre l'ensemble du problème de la reconstruction d'image en tomographie. Mais nous nous intéressons seulement à la partie "synthèse de Fourier " du problème c'est-à-dire au problème de l'utilisation d'une projection ou de sa transformée de Fourier (TF), une fois que sa relation avec la TF 2-D de l'objet a été fixée, et sans se préoccuper des approximations que cette relation peut cacher (ex: Born, Rytov etc.). Cette partie est commune à beaucoup de techniques d'imagerie, que ce soit en tomographie à rayons $\mathrm{X}$ ou en tomographie à onde diffractée ou bien même en imagerie RMN.

Pour ceci nous allons considérer une fonction 2-D $f(x, y)$. Nous avons une information partielle $\hat{f}(\beta, \alpha)$ sur $\hat{f}(u, v)$, où $(\beta, \alpha)$ sont reliées aux coordonnées cartésiennes $(u, v)$ par une transformation $T$ :

$$
\hat{f}(u, v)=\hat{f}(\beta, \alpha)
$$

où

$$
[u, v]=T(\beta, \alpha)=\left[T_{1}(\beta, \alpha), T_{2}(\beta, \alpha)\right]
$$

et où $T_{1}$ et $T_{2}$ sont supposées être continues. Les lieux des points définis par $T$ forment un ensemble de contours dans le domaine de Fourier [2, 7].

Dans le cas de la tomographie à onde diffracté nous avons

$$
\left(\begin{array}{l}
u \\
v
\end{array}\right)=\left(\begin{array}{rr}
\cos \theta & -\sin \theta \\
\sin \theta & \cos \theta
\end{array}\right)\left(\begin{array}{l}
\beta \\
\alpha
\end{array}\right)=\left(\begin{array}{l}
T_{1}(\theta, \alpha) \\
T_{2}(\theta, \alpha)
\end{array}\right)
$$

où

$$
\beta=-k_{0}+\left(k_{0}^{2}-\alpha^{2}\right)^{1 / 2}
$$

Dans ce cas les contours définis par $T$ sont des demicercles de rayons $k_{0}$ [(cf. Fig. (3)]. On obtient, par TF inverse

$$
\begin{aligned}
f(x, y)=\iint \hat{f}(\theta, \alpha) J(\theta, \alpha) \times \\
\quad \times \exp \left\{j\left[T_{1} x+T_{2} y\right]\right\} \mathrm{d} \theta \mathrm{d} \alpha
\end{aligned}
$$


où $J(\theta, \alpha)$ est le jacobien donné par

$$
J(\theta, \alpha)=\left(\begin{array}{ll}
\partial T_{1} / \partial \theta & \partial T_{1} / \partial \alpha \\
\partial T_{2} / \partial \theta & \partial T_{2} / \partial \alpha
\end{array}\right)
$$

Il n'est pas difficile de montrer que dans le cas de la tomographie à rayons $\mathrm{X}$ on a $J(\theta, \alpha)=|\alpha|$ et, que dans le cas de la tomographie à onde diffractée on a $J(\theta, \alpha)=k_{0}|\alpha| /\left(k_{0}^{2}-\alpha^{2}\right)^{1 / 2}$.

L'équation (3) et sa TF inverse montrent que l'on peut développer deux types de méthodes d'interpolation suivant l'ordre des intégrations.

2.1.1 Interpolation dans le domaine de Fourier (Focalisation synthétique). - Dans cette méthode on fait l'hypothèse que l'objet est à support borné, et donc que sa TF est analytique. Soit

$$
I(x, y)= \begin{cases}1 & (x, y) \in \mathrm{D} \\ 0 & (x, y) \notin \mathrm{D}\end{cases}
$$

et sa TF

$$
\hat{I}(u, v)=\iint_{\mathrm{D}} \exp [-j(u x+v y)] \mathrm{d} x \mathrm{~d} y
$$

La TF inverse de l'équation (3) peut être écrite

$$
\begin{aligned}
\hat{f}(u, v)=\iint & \hat{f}(\theta, \alpha) J(\theta, \alpha) \times \\
& \times \iint \exp \left[-j\left(\left(u-T_{1}\right) x+\left(v-T_{2}\right) y\right)\right] \\
& \times \mathrm{d} x \mathrm{~d} y \mathrm{~d} \theta \mathrm{d} \alpha
\end{aligned}
$$

ou bien

$$
\begin{aligned}
\hat{f}(u, v)=\iint \hat{f}(\theta, \alpha) J(\theta, \alpha) \times & \\
& \times \hat{I}\left[\left(u-T_{1}\right),\left(v-T_{2}\right)\right] \mathrm{d} \theta \mathrm{d} \alpha .
\end{aligned}
$$

Ainsi l'algorithme de reconstruction est le suivant :

1) calcul de $\hat{f}(\theta, \alpha)$ à partir de mesures du champ diffracté,

2) interpolation pour obtenir $\hat{f}(u, v)$ à l'aide de l'équation (6),

3) calcul de la TF inverse de $\hat{f}(u, v)$ pour obtenir $f(x, y)$.

En pratique on ne connaît $\hat{f}(\theta, \alpha)$ que pour des valeurs discrètes et en nombre fini de $\theta_{m}$ et de $\alpha_{n}$ et on doit faire d'autres hypothèses pour discrétiser cette équation intégrale. Par exemple par souci de simplicité on fait l'hypothèse que l'on peut écrire

$$
\begin{aligned}
\hat{f}(\theta, \alpha)=\sum \sum \hat{f}\left(\theta_{m}, \alpha_{n}\right) \times & \\
& \times \delta\left(\theta-\theta_{m}\right) \delta\left(\alpha-\alpha_{n}\right) .
\end{aligned}
$$

Ceci veut dire que l'on suppose que les données manquantes sont nulles, ce qui simplifie bien les choses mais ce qui est une hypothèse brutale. Dans ce cas on a

$$
\begin{aligned}
\hat{f}(u, v)= & \sum_{m} \sum_{n} \hat{f}\left(\theta_{m}, \alpha_{n}\right) J\left(\theta_{m}, \alpha_{n}\right) \times \\
& \times \hat{I}\left[u-T_{1}\left(\theta_{m}, \alpha_{n}\right), v-T_{2}\left(\theta_{m}, \alpha_{n}\right)\right] .
\end{aligned}
$$

On peut encore simplifier cette équation en se limitant au lobe principal de la fonction $\hat{I}(u, v)$ :

$$
\begin{aligned}
\hat{f}(u, v)= & \sum_{m, n \in \text { lobe principal de } \hat{I}} \hat{f}\left(\theta_{m}, \alpha_{n}\right) J\left(\theta_{m}, \alpha_{n}\right) \times \\
& \times \hat{I}\left[u-T_{1}\left(\theta_{m}, \alpha_{n}\right), v-T_{2}\left(\theta_{m}, \alpha_{n}\right)\right] .
\end{aligned}
$$

Une simplification plus importante peut être faite, en limitant le domaine de l'interpolation à un point, chaque point étant remplacé par la valeur de son voisin le plus proche (interpolation d'ordre zéro) $[6,8]$.

2.1.2 Interpolation dans le domaine spatial (Rétropropagation). - L'hypothèse retenue est ici duale de l'hypothèse précédente, on suppose que le spectre de l'objet est à support borné, et donc que l'objet est analytique.

Définissons les coordonnées $\left(x^{\prime}, y^{\prime}\right)$ telles que

$$
\begin{aligned}
T_{1} x+T_{2} y=[x, y]\left(\begin{array}{l}
T_{1} \\
T_{2}
\end{array}\right) & = \\
= & {[x, y]\left(\begin{array}{rr}
\cos \theta-\sin \theta \\
\sin \theta & \cos \theta
\end{array}\right)\left(\begin{array}{l}
\beta \\
\alpha
\end{array}\right) } \\
& =\left[x^{\prime}, y^{\prime}\right]\left(\begin{array}{l}
\beta \\
\alpha
\end{array}\right)=x^{\prime} \beta+y^{\prime} \alpha .
\end{aligned}
$$

Définissons aussi une nouvelle fonction $I_{\theta}\left(x^{\prime}, y^{\prime}\right)$ appelée «fonction rétropropagée » [2], par

$$
\begin{aligned}
I_{\theta}\left(x^{\prime}, y^{\prime}\right)=\int \hat{f}(\theta, \alpha) J(\theta, \alpha) \exp \left(j \beta x^{\prime}\right) \times \\
\times \exp \left(j \alpha y^{\prime}\right) \mathrm{d} \alpha \\
=\mathrm{TF}^{-1}\left\{\hat{f}(\theta, \alpha) J(\theta, \alpha) \exp \left(j \beta x^{\prime}\right)\right\} . \quad(10)
\end{aligned}
$$

Ainsi l'équation (22) peut être écrite

$$
\begin{aligned}
& f(x, y)= \\
& \quad=\int I_{\theta}(x \cos \theta+y \sin \theta,-x \sin \theta+y \cos \theta) \mathrm{d} \theta .
\end{aligned}
$$

L'algorithme de reconstruction est donc le suivant :

1) Calcul de $\hat{f}(\theta, \alpha)$ à partir de mesures du champ diffracté.

2) Calcul de $I_{\theta}\left(x^{\prime}, y^{\prime}\right)$, par une TF inverse de $\hat{f}(\theta, \alpha) J(\theta, \alpha) \exp \left(j, \beta x^{\prime}\right)$, ceci pour les différentes valeurs de $x^{\prime}$.

3) Interpolation dans le domaine spatial.

Ici aussi on doit faire d'autres hypothèses pour discrétiser ces équations intégrales. Ce sont par exemple les mêmes hypothèses brutales que dans l'équation (7) [8] 
2.2 DifFICUltÉS THÉORIQUES. - Le problème auquel nous sommes confrontés est celui de la synthèse de Fourier, c'est-à-dire celui de la reconstruction d'une fonction à support borné $f(x, y)$, connaissant un nombre fini de points de sa transformée de Fourier. Une question clé soulevée ici, et d'ailleurs dans n'importe quel problème de reconstruction, est la suivante : Le problème est-il bien posé ? Pour qu'un problème soit bien posé sa solution doit satisfaire les deux conditions usuelles d'existence et d'unicité et la condition physique de stabilité.

Les méthodes de reconstruction discutées dans le paragraphe précédent effectuent une interpolation, soit dans le domaine de Fourier en faisant l'hypothèse d'un support borné de la fonction dans le domaine spatial, soit dans le domaine spatial en faisant l'hypothèse d'un support borné du spectre de la fonction.

En tomographie à rayons $\mathrm{X}$, les contours sur lesquels les données sont disponibles sont des lignes droites ; en tomographie à onde diffractée ces contours sont des demi-cercles. Mais dans tous les cas ces contours sont obtenus à partir d'une transformation connue des coordonnées cartésiennes. Aussi dans les deux cas les données sur une ligne ou sur un demi-cercle dans le domaine de Fourier sont obtenues par la TF d'une projection ou du champ diffracté mesuré sur une ligne de mesure.

Mais il est important de noter qu'en général un signal multidimensionnel $f$ à bande limitée $(\hat{f}(\omega)=0$, $\omega \notin \Omega)$ ne peut être reconstruit d'une façon unique à partir d'un nombre fini et même d'une continuité d'échantillons de sa TF sur un contour algébrique [9].

Ainsi on peut dire qu'un nombre fini de projections ou de lignes de mesures du champ diffracté (même si on avait des mesures continues) ne permet pas de reconstruire l'objet d'une façon unique, sauf si on ajoute d'autres informations a priori sur l'objet. Mais Sanz [10] a montré que même en imposant une norme de douceur sur le spectre de l'objet à reconstruire (ceci jouant un rôle important dans beaucoup d'autres problèmes de restauration et de reconstruction), on ne réduit pas l'incertitude de ce problème.

Ce point surprenant vient de ce que beaucoup de résultats valables sur la droite ne sont pas généralisables au plan.

A ce manque d'unicité de la solution dans la situation idéale d'une infinité de mesures vient se rajouter la difficulté supplémentaire des erreurs de mesure dont il n'est pas tenu compte dans ces méthodes déterministes.

\subsection{DifFICULTÉS PRATIQUES DE MISE EN GEUVRE. -} Les algorithmes de reconstruction existants nécessitent par leurs hypothèses explicites et implicites qu'on dispose d'un nombre suffisamment grand de projections uniformément distribuées autour de l'objet à reconstruire. En pratique et dans beaucoup de circonstances ceci n'est pas réalisable. Généralement on ne dispose que d'un nombre limité de projections, qui ne sont pas nécessairement distribuées d'une façon uniforme autour de l'objet. De plus elles sont aussi limitées par la longueur de la ligne de mesure et le nombre de points de mesure sur cette ligne.

Le résultat de ces contraintes expérimentales est que l'espace de Fourier de la fonction à reconstituer n'est pas rempli de façon assez dense ou bien ce remplissage n'est que sectoriel (Fig. 4). Par conséquent, les méthodes discutées plus haut ne donnent plus de résultats satisfaisants et peuvent même induire des erreurs.

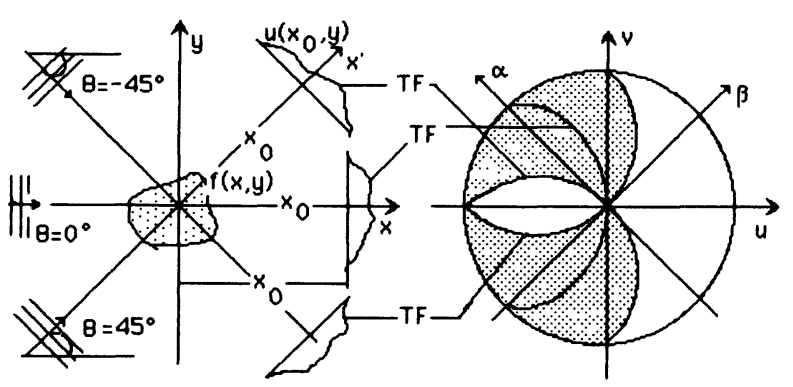

Fig. 4. - Remplissage incomplet du domaine de Fourier en tomographie à onde diffractée dans le cas où les projections sont limitées par des obstacles $\left(\theta \in\left[-45^{\circ} \grave{a}+45^{\circ}\right]\right)$.

[Incomplete filling of the Fourier domain in diffraction tomography in the case of a limitation of the projections by obstacles $\left(\theta \in\left[-45^{\circ},+45^{\circ}\right]\right)$.

Dans le paragraphe suivant un certain nombre de résultats de simulations sont présentés qui mettent en évidence ces difficultés.

2.4 Résultats De Simulation. - Dans les exemples présentés, nous nous sommes placés dans le cadre de l'approximation de Born et, par simulation, nous avons calculé le champ diffracté en discrétisant l'équation intégrale (13) ; ceci pour les différentes valeurs de l'angle de rotation $\theta_{i}$. Ensuite nous avons calculé la TFD de ces champs et l'avons multipliée par des termes de propagation (Eq. 18), pour obtenir les valeurs de $f\left(\theta_{m}, \alpha_{n}\right)$. Et finalement nous avons reconstitué l'objet $f(x, y)$ par les deux méthodes de reconstruction suivantes :

M1) méthode de reconstruction par focalisation synthétique et,

M2) méthode de reconstruction par rétropropagation.

Nous avons effectué ce travail pour trois types d'objet :

O1) Un objet ponctuel pour mettre en évidence la réponse impulsionnelle du processus de reconstruction,

O2) un objet cylindrique pour mettre en évidence sa réponse indicielle,

O3) un objet composé de quatre cylindres avec les amplitudes différentes et disposés d'une façon quelconque pour éliminer toute redondance due à une symétrie de révolution (Fig. 5A). 


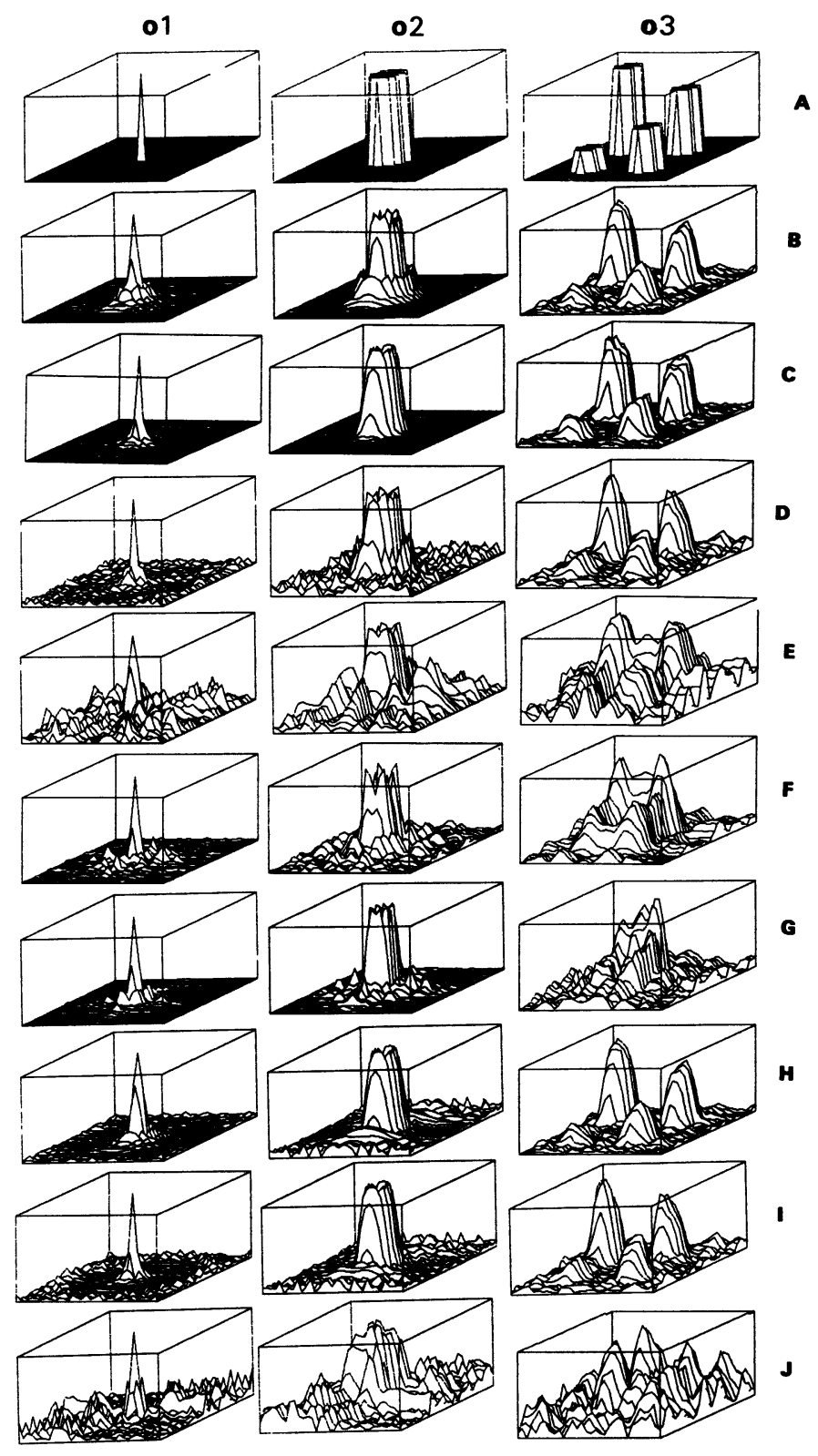

Fig. 5. - Résultats de simulation de trois objets différents $\mathrm{O} 1, \mathrm{O} 2$ et $\mathrm{O} 3$ par les méthodes de reconstructions classiques (M1 : interpolation dans le domaine de Fourier, M2 : interpolation dans le domaine spatial): a) objets $\mathrm{O} 1, \mathrm{O} 2$ et $\mathrm{O} 3$, b) M1, $N_{\mathrm{P}}=\infty, L=8 \lambda$, c) M1, $N_{\mathrm{P}}=\infty, L=16 \lambda$, d) M1, $N_{\mathrm{P}}=32, L=16 \lambda$, e) $\mathrm{M} 1, N_{\mathrm{P}}=4, L=16 \lambda$, INTP $=0$, f) M1, $N_{\mathrm{P}}=4, L=16 \lambda, \quad$ INTP $=1, L_{\mathrm{P}}=1$, g) M1, $N_{\mathrm{P}}=4, L=16 \lambda, \quad$ INTP $=1, L_{\mathrm{P}}=4$, h) M2, $N_{\mathrm{P}}=32, L=8 \lambda$, i) $\mathrm{M} 2, \quad N_{\mathrm{P}}=32, L=16 \lambda$, j) $\mathrm{M} 2$, $N_{\mathrm{P}}=4, L=16 \lambda$.

[Reconstruction results of 3 different objects $\mathrm{O} 1, \mathrm{O} 2$ and $\mathrm{O} 3$ by classical methods of reconstruction (M1 : Fourier domain interpolation, M2 : Spatial domain interpolation) : a) objects $\mathrm{O} 1, \mathrm{O} 2$ and $\mathrm{O} 3$, b) $\mathrm{M} 1, N_{\mathrm{P}}=\infty, L=8 \lambda$, c) $\mathrm{M} 1$, $N_{\mathrm{P}}=\infty, L=16 \lambda$, d) M1, $N_{\mathrm{P}}=32, L=16 \lambda$, e) M1, $N_{\mathrm{P}}=4, L=16 \lambda$, INTP $=0$, f) $\mathrm{M} 1, N_{\mathrm{P}}=4, L=16 \lambda$, INTP $\left.=1, L_{\mathrm{P}}=1, \mathrm{~g}\right) \mathrm{M} 1, N_{\mathrm{P}}=4, L=16 \lambda$, INTP $=1$, $L_{\mathrm{P}}=4$, h) M2, $N_{\mathrm{P}}=32, L=8 \lambda$, i) $\mathrm{M} 2, N_{\mathrm{P}}=32, L=$ $16 \lambda$, j) M2, $N_{\mathrm{P}}=4, L=16 \lambda$.]
Nous avons organisé ces résultats pour séparer les effets des différents paramètres sur la qualité de la reconstruction.

Dans ces figures

$-N_{\mathrm{p}}$ est le nombre de projections,

$-L$ est la longueur de la ligne de mesure,

$-\lambda$ est la longueur d'onde,

- INTP est l'ordre d'interpolation et,

- $L_{\mathrm{p}}$ est la longueur du domaine d'interpolation.

\section{C)}

Effet de la longueur de la ligne de mesure (Figs. 5B et

Méthode $\mathrm{M} 1, N_{\mathrm{p}}=\infty$.

B) Résultats de reconstruction avec $L=8 \lambda$.

C) Résultats de reconstruction pour $L=16 \lambda$.

On constate l'effet de la troncature due à la longueur de la ligne de mesure et sa conséquence néfaste sur le calcul de sa TF.

Effet du nombre de projections (Figs. 5C, D et E) Méthode M1, $L=16 \lambda$.

C) Résultats de reconstruction avec $N_{\mathrm{p}}=\infty$.

D) Résultats de reconstruction avec $N_{\mathrm{p}}=32$.

E) Résultats de reconstruction avec $N_{\mathrm{p}}=4$.

Effet du choix de l'interpolation (Figs. 5E, F et G) Méthode M1, $N_{\mathrm{p}}=4$ et $L=16 \lambda$.

E) Interpolation d'ordre zéro (INTP $=0$, voisin le plus proche).

F) Interpolation d'ordre un en limitant le domaine de l'interpolation à la longueur du lobe principal $\left(\right.$ INTP $\left.=1, L_{\mathrm{p}}=1\right)$.

G) Interpolation d'ordre un en limitant le domaine de l'interpolation à 4 fois la longueur du lobe principal $\left(\right.$ INTP $\left.=1, L_{\mathrm{p}}=4\right)$.

J)

Comparaison entre les deux méthodes (Figs. 5H, I et

Méthode M2 (rétropropagation)

H) $L=8 \lambda, N_{\mathrm{p}}=32$ (à comparer avec B).

I) $L=16 \lambda, N_{\mathrm{p}}=32$ (à comparer avec C).

J) $L=16 \lambda, N_{\mathrm{p}}=4$ (à comparer avec D).

2.5 ConClusions. - Les résultats de simulation confirment les conclusions qui se dégagent de la comparaison des principes des méthodes de reconstruction existantes.

i) Les difficultés majeures de ces méthodes sont à deux niveaux :

- au passage dans le domaine de Fourier, à cause de la limitation du support et du nombre de points de mesure du champ diffracté,

- au retour dans le domaine spatial, à cause des interpolations effectuées pour remplir le domaine de Fourier.

ii) Dans des conditions idéales, c'est-à-dire un nombre de projections très grand, une longueur des lignes de mesure très grande, et des mesures sans bruit, les 
deux méthodes de reconstruction 1 et 2 fournissent des résultats analogues et de bonne qualité.

iii) Dans des conditions défavorables, c'est-à-dire un nombre de projections faible ou resteint à un angle limité, une longueur de la ligne de mesure réduite, il est difficile de comparer ces méthodes. Il est d'ailleurs très difficile de faire confiance aux résultats alors obtenus car le choix des paramètres de l'interpolation joue un rôle important dans les résultats et ce choix ne peut être fait que d'une façon empirique.

\section{Méthode de reconstruction par maximum d'entropie.}

3.1 DÉFINITIONS ET POSITION DU PROBLÈME. Dans une situation défavorable (limitation du nombre de vues, du nombre de points de mesure, de l'étendue du segment de mesure), le principal défaut des méthode exposées tient dans les hypothèses $a$ priori faites sur l'objet. Quand elles ne sont pas implicites (interpolation), elles sont faites plus par commodité que par conviction. La situation est en fait celle où les données observées sont largement insuffisantes pour déterminer la solution. Il nous semble que l'on gagnerait à reconnaître cette situation et à l'affronter avec des méthodes d'inférence plus cohérentes.

Le principe du maximum d'entropie (ME) nous semble un bon candidat pour développer une méthode de reconstruction d'images à partir de données incomplètes et bruitées.

L'utilisation de ce principe repose sur l'assignation d'une mesure d'entropie pour caractériser le caractère incertain ou aléatoire de l'objet dans son environnement statistique. Chaque mesure effectuée ou chaque élément d'une information a priori, en supposant qu'ils soient sans contradiction entre eux, diminue le caractère aléatoire et les degrés de liberté du problème. Si les données sont insuffisantes pour éliminer tout le caractère incertain des paramètres à estimer, ce principe garantit que la solution fournie n'est que le reflet de la description incomplète du problème, c'est-à-dire que l'on ne va pas ajouter d'information où il n'y en a pas.

Il existe deux écoles de pensée pour la définition de l'entropie d'une image. Nous les appelons par « $\log X$ » [12] et «-X $\log X$ » $[13,15]$.

Johnson et Shore [11] ont montré que ces deux expressions sont les résultats de l'application du principe plus général de minimisation de l'entropie croisée. Ce principe nous permet d'estimer une fonction de densité de probabilité (FDP) $q(\mathbf{x})$, connaissant une densité a priori $p(\mathbf{x})$ et des données $m_{i}$ sous forme de

$$
\int q(\mathbf{x}) g(\mathbf{x}) \mathrm{d} \mathbf{x}=m_{i} \quad i=1, \ldots, M .
$$

Cette estimée est obtenue en minimisant l'entropie croisée $H(p, q)$ définie par

$$
H(q, p)=\int q(\mathbf{x}) \log [q(\mathbf{x}) / p(\mathbf{x})] \mathrm{d} \mathbf{x}
$$

sous les contraintes (1) et la contrainte de normalisation :

$$
\int q(\mathbf{x}) \mathrm{d} \mathbf{x}=1
$$

Le résultat de cette estimation a la forme de

$$
q(\mathbf{x})=p(\mathbf{x}) \exp \left[-\alpha-\sum \beta_{i} m_{i}\right]
$$

où $\alpha$ et $\beta_{i}$ sont des multiplicateurs de Lagrange déterminés par (3) et (1). La minimisation de l'entropie croisée se réduit à la maximisation de l'entropie « $-X \log X »$ si l'a priori $p(\mathbf{x})$ est uniforme.

Kikuchi et Soffer [14] ont aussi montré, par une approche quantique, qu'il s'agissait en fait de deux approximations différentes d'une même mesure d'information, correspondant à des situations physiques différentes.

En analyse spectrale par ME, l'entropie est définie soit par

$$
H_{1}=\int \log S(\omega) \mathrm{d} \omega
$$

soit par

$$
H_{2}=-\int S(\omega) \log S(\omega) \mathrm{d} \omega .
$$

La différence entre ces deux expressions est dans les quantités qui sont traitées dans le problème comme des variables aléatoires (v.a.). Dans la première expression, les v.a. concernées sont les coefficients de la décomposition en série de Fourier du signal, et les $S(\omega)$ sont leurs espérances mathématiques. Dans la deuxième expression, $S(\omega)$, normalisée convenablement, est traitée comme une densité de probabilité et, les v.a. concernées sont des fréquences.

Si le processus d'imagerie peut être considéré comme un problème d'estimation spectrale à $2-\mathrm{D}$, on peut alors définir l'entropie soit par

$$
H_{1}=\iint \log f(x, y) \mathrm{d} x \mathrm{~d} y
$$

ou par

$$
H_{2}=-\iint f(x, y) \log f(x, y) \mathrm{d} x \mathrm{~d} y
$$

où $f(x, y)$ désigne la fonction caractérisant l'objet que l'on cherche à reconstruire. Ceci est le cas au moins dans un domaine important d'application des méthodes de reconstruction d'image. C'est la radio-astronomie où une source radio incohérente crée un champ électrique aléatoire dont la fonction de corrélation spatiale peut être mesurée par interférométrie. La relation entre la distribution de l'intensité de la source et les mesures obtenues par les interféromètres est une relation de TF. 
Pour un problème de tomographie à onde diffractée, si, dans un premier temps, on fait abstraction des difficultés liées au passage du domaine des mesures au domaine de Fourier, le problème de reconstruction peut se formuler ainsi :

Estimer au mieux $f(x, y)$ connaissant un nombre fini d'échantillons de sa TF 2-D

$$
\begin{aligned}
m_{i}=\iint f(x, y) \exp [-j(u x+v y)] \mathrm{d} x \mathrm{~d} y & \\
& \quad i=1, \ldots, M .
\end{aligned}
$$

Les méthodes directes de reconstruction, c'est-à-dire les méthodes dans lesquelles on suppose que les données manquantes sont nulles, ne donnent des résultats satisfaisants que si les données couvrent bien l'espace de Fourier sur des coordonnées cartésiennes $(u, v)$. Dans le cas contraire il faut inclure une information a priori pour trouver une solution unique et acceptable. La difficulté est alors de savoir comment introduire cette information et de vérifier si elle garantit l'existence, l'unicité et la stabilité de la solution, ce qui n'est pas le cas pour n'importe quelle information $a$ priori.

Le concept de maximum d'entropie assure une reconstruction unique et positive, si les mesures sont sans bruit, car l'entropie $H$ diverge si un nombre important de points de la solution tendent vers zéro.

Une reconstruction à $\mathrm{ME}$ pourrait donc être définie comme le problème d'optimisation suivant :

Maximiser $H(f)=\iint \log f(x, y) \mathrm{d} x \mathrm{~d} y$ sous les contraintes

$$
\begin{aligned}
& \iint f(x, y) \exp [-j(u x+v y)] \mathrm{d} x \mathrm{~d} y=m_{i} \\
& i=1, \ldots, M
\end{aligned}
$$

et,

$$
f(x, y)>0 \quad \text { si } \quad(x, y) \in D .
$$

Il n'existe pas de solution explicite à ce problème et l'existence de la solution n'est pas toujours assurée. De plus, on ne tient pas compte du bruit sur les observations.

Dans les paragraphes suivants nous allons considérer le problème de la reconstruction sous forme discrète et, par la nécessité d'une application au problème de la tomographie à onde diffractée où l'image à reconstruire représente une distribution de grandeurs complexes, nous allons définir tout d'abord l'entropie d'une image complexe, ensuite nous discuterons le problème de la prise en compte du bruit sur les données, et finalement nous présenterons un algorithme de mise en œuvre et des résultats d'application.

3.2 DÉFINITION DE L'ENTROPIE DANS LE CAS COMPLEXE. - Dans le cas de la reconstruction d'image on suppose que l'image est partitionnée en $N$ pixels et que la valeur de chaque pixel est représentée par un nombre réel positif $f_{n}$. En imagerie optique ou en astronomie l'image à reconstruire est une grandeur réelle et positive, car il s'agit de l'intensité ou du nombre de photons émis (ou absorbés) par l'objet. Mais dans le cas de l'imagerie micro-onde la grandeur à estimer peut être soit une grandeur réelle, soit une grandeur complexe. C'est pourquoi nous allons considérer les deux cas suivants :

3.2.1 $f_{n}$ est une grandeur réelle. - On définit alors l'entropie de l'image $\mathbf{f}=\left[f_{1}, \ldots, f_{n}, \ldots, f_{N}\right]$, soit par

$$
H_{1}(\mathbf{f})=\sum \log f_{n}
$$

ou par

$$
H_{2}(\mathbf{f})=-\sum f_{n} \log f_{n}
$$

3.2.2 $f_{n}$ est une grandeur complexe $f_{n}=$ $\left(f_{n}^{\mathrm{R}}+j f_{n}^{\mathrm{I}}\right)$. - Dans ce cas on peut définir $H(\mathbf{f})$ des deux façons suivantes :

a) Si la partie réelle $f_{n}^{\mathrm{R}}$ et la partie imaginaire $f_{n}^{\mathrm{I}}$ peuvent être considérées comme deux variables positives et indépendantes (ce qui est le cas de la permittivité complexe en tomographie micro-onde par exemple quand la fréquence est constante) on définit $H(\mathbf{f})$ soit par

$$
H(\mathbf{f})=\sum\left(\log f_{n}^{\mathrm{R}}+\log f_{n}^{\mathrm{I}}\right)
$$

soit par

$$
H(\mathbf{f})=-\sum\left(f_{n}^{\mathrm{R}} \log f_{n}^{\mathrm{R}}+f_{n}^{\mathrm{I}} \log f_{n}^{\mathrm{I}}\right) .
$$

b) Si par contre on s'intéresse à l'amplitude de $f_{n}$, ce qui peut être le cas de la densité de courant induite à l'intérieur de l'objet diffractant en tomographie micro-onde, on définit $H(\mathbf{f})$ soit par

$$
H(\mathbf{f})=\sum\left(\log \left|f_{n}\right|\right)
$$

soit par

$$
H(\mathbf{f})=-\sum\left(\left|f_{n}\right| \log \left|f_{n}\right|\right)
$$

3.3 PRISE EN COMPTE DU BRUIT SUR LES DONNÉES. - Les mesures physiques ne sont jamais sans erreur et l'information sur la statistique du bruit de mesure constitue une information $a$ priori très importante qui doit être incluse dans la formulation du problème. Pour ceci nous allons redéfinir le problème d'une façon plus générale en supposant que l'objet a été discrétisé et que les mesures sont entachées de bruit. Supposons que les données mesurées sont reliées à l'objet à reconstruire $f$ par

$$
m_{i}=\sum A_{\text {in }} f_{n}+b_{i} \quad i=1, \ldots, M
$$

ou bien sous forme matricielle $\mathbf{m}=A \mathbf{f}+\mathbf{b}$ où les $b_{i}$ sont des termes de bruit avec une distribution gaussienne $N\left(0, \sigma_{i}\right)$. Pour pouvoir prendre en compte le bruit, nous allons modifier les contraintes des 
observations en se plaçant dans le contexte d'une statistique du $\chi^{2}$. Pour mesurer le désaccord entre les données mesurées $m_{i}$ (bruitées) et les données simulées $\hat{m}_{i}$ qui auraient été obtenues si l'image était représentée par $\mathbf{f}$ en absence du bruit, nous définissons le critère quadratique suivant

$$
Q(\mathbf{f})=\sum\left(1 / \sigma_{i}^{2}\right)\left|m_{i}-\hat{m}_{i}\right|^{2} \quad \text { où } \quad \hat{m}_{i}=\sum A_{i n} f_{n}
$$

ou bien encore, sous forme matricielle

$$
Q(\mathbf{f})=[A \mathbf{f}-\mathbf{m}]^{t} D[A \mathbf{f}-\mathbf{m}]
$$

où

$$
D=\operatorname{diag}\left[1 / \sigma_{1}^{2}, \ldots, 1 / \sigma_{M}^{2}\right]
$$

Bien entendu d'autres choix pour $Q(\mathbf{f})$ sont possibles et même préférables dans d'autres circonstances [13].

Il est évident qu'il est plus facile d'utiliser et de satisfaire une seule contrainte statistique que d'essayer de satisfaire chacune des contraintes sur les mesures.

Ainsi nous sommes devant le problème d'optimisation suivant: Maximiser $H(\mathbf{f})$ sous la contrainte $Q(\mathbf{f}) \leqslant M, M$ étant le nombre des observations.

Ce problème peut être résolu par une méthode variationnelle, c'est-à-dire qu'on définit une fonction lagrangienne $J(\mathbf{f})=Q(\mathbf{f})-\lambda Q(\mathbf{f})$ et la solution se trouve sur un extrémum de $J(\mathbf{f})$ pour une valeur définie de $\lambda$. $\lambda$ joue le rôle d'un multiplicateur de Lagrange. La maximisation de $J(\mathbf{f})$ peut être considérée comme un effort pour à la fois maximiser l'entropie et minimiser l'erreur moyenne quadratique résiduelle avec une importance relative donnée par le paramètre $\lambda[8,12]$.

La contrainte $Q(\mathbf{f}) \leqslant M$ résultant de l'application du théorème de la limite centrale (statistique du $\chi^{2}$ ) détermine un ensemble de solutions possibles $\hat{\mathbf{f}}$ qui est consistant statistiquement avec les données mesurées. Parmi ces solutions on choisit la solution qui maximise l'entropie et on montre qu'elle est unique [12].

A ce stade on peut aussi donner une interprétation différente de cette méthode. En effet la maximisation de $J(f)$ peut être interprétée comme la recherche d'une solution régularisée à l'équation (10) par une technique de moindres carrés (MC) sous contrainte dans laquelle $H(f)$ est la fonction de régularisation. Dans cette interprétation, il faut remarquer que cette fonction est fortement non linéaire. C'est justement cette nonlinéarité qui apporte une amélioration de résolution par rapport aux méthodes d'interpolation linéaires.

Il est à noter que si $\mathbf{f}\left(f_{n}>0\right)$ est une solution de ce problème, il satisfait l'équation suivante

$$
\nabla J(\mathbf{f})=\left[\partial J(\mathbf{f}) / \partial f_{1}, \ldots, \partial J(\mathbf{f}) / \partial f_{n}\right]=\mathbf{0}
$$

où

$$
\begin{aligned}
& {\left[\partial J(\mathbf{f}) / \partial f_{1}, \ldots, \partial J(\mathbf{f}) / \partial f_{n}\right]=} \\
& \quad=\left[1 / f_{1}, \ldots, 1 / f_{n}\right]-\lambda A^{\mathrm{t}} D[A \mathbf{f}-\mathbf{m}]
\end{aligned}
$$

et où

$\nabla^{2} J(\mathbf{f})=-\operatorname{diag}\left[1 / f_{1}^{2}, \ldots, 1 / f_{n}^{2}\right]-\lambda A^{\mathrm{t}} D A<0$

$$
\text { si } \lambda>0
$$

est une matrice définie négative. Ainsi $J(\mathbf{f})$ est une fonction strictement concave et on démontre [12] que si $\lambda>0$ l'équation (13) ne peut avoir qu'une solution, et cette solution, si elle existe, est le maximum de $J(\mathbf{f})$.

3.4 MÉTHOdES D’OPTIMISATION. - L'entropie étant intrinsèquement non linéaire, le problème est donc celui d'une optimisation sous contrainte. En imagerie le problème est aussi de grandes dimensions et par conséquence il n'est pas possible d'utiliser des méthodes du second ordre comme les techniques itératives de Newton-Raphson, utilisées sur des problèmes de petites dimensions. Dans ce paragraphe nous allons brièvement exposer les différentes méthodes utilisées dans des problèmes analogues.

3.4.1 Résolution d'équation intégrale. - Gull et Daniell [15] ont essayé de trouver une solution à l'équation $(\nabla J(f)=0)$ en maximisant directement $J(\mathbf{f})=H(\mathbf{f})-\lambda Q(\mathbf{f})$ pour une valeur fixe de $\lambda$. Ils ont utilisé la forme itérative suivante :

$$
f_{n}^{(k+1)}=Z \exp \left[-\lambda \partial Q\left(\mathbf{f}^{(k)}\right) / \partial f_{n}\right]
$$

où $(k)$ indique la $k$-ième itération. Cette procédure a l'avantage intéressant de garantir à chaque itération la positivité de la solution. Mais malheureusement l'algorithme est instable et l'introduction d'un coefficient de stabilisation sous forme de

$$
\begin{aligned}
f_{n}^{(k+1)}=(1-\alpha) f_{n}^{(k)} & + \\
& +\alpha \exp \left[-\lambda \partial Q\left(\mathbf{f}^{(k)}\right) / \partial f_{n}\right]
\end{aligned}
$$

ne permet pas de stabiliser la solution. Cependant leur travail est d'une importance cruciale pour montrer la possibilité d'obtenir des images de haute résolution.

3.4.2 Méthodes du gradient. - L'approche la plus simple pour maximiser $J(\mathbf{f})$ est d'utiliser

$$
f_{n}^{(k+1)}=f_{n}^{(k)}+\alpha \partial Q\left(\mathbf{f}^{(k)}\right) / \partial f_{n}
$$

pour une valeur appropriée de $\alpha$. L'inconvénient majeur de cette technique est que, pour des valeurs grandes de $\alpha$, il peut y avoir des valeurs négatives de $\partial Q(\mathbf{f}) / \partial f_{n}$ qui rendent négatives les valeurs de $f_{n}^{(n+1)}$ à l'itération suivante. D'autre part la méthode ne converge pas rapidement.

La procédure standard pour améliorer les performances de l'algorithme du gradient consiste à utiliser la technique du gradient conjugué de Flechter et Reeves [16], ou une variante de celle-ci [17]. Le principe est qu'à chaque itération, au lieu d'utiliser $\nabla J(f)$ comme direction dans laquelle on effectue la recherche du maximum de $J(\mathbf{f})$, on utilise seulement une partie 
$\mathbf{e}^{(n)}$ de $\nabla J(\mathbf{f})$ qui est conjuguée des directions précédentes $\left(\mathbf{e}^{(r)}, r=1, \ldots, n-1\right)$ définie par

$$
\left(\mathbf{e}^{(n) t} \cdot \nabla J \cdot \mathbf{e}^{(r)}=0, r=1, \ldots, n-1\right) .
$$

Autrement dit on cherche le maximum du $\nabla J$ sur les points $\mathbf{f}^{(n)}+\alpha \mathbf{e}^{(n)}$ se trouvant sur les directions de recherches $\mathbf{e}^{(n)}$ où $\mathbf{e}^{(n)}=-\nabla J+\beta \mathbf{e}^{(n-1)}$ avec $\beta=\left|\nabla J^{(n)}\right|^{2} /\left|\nabla J^{(n-1)}\right|^{2}$ ou une forme équivalente à celle-ci. Les coefficients $\beta$ sont définis en faisant l'hypothèse que $J(f)$ a une courbure constante. Ceci n'est pas réellement vrai dans notre cas où une des composantes de $J(\mathbf{f})$, c'est-à-dire $H(\mathbf{f})$, est non quadratique, mais permet cependant d'accélérer sensiblement la convergence.

Cette méthode a été utilisée par Wernecke et d'Addario [12], pour un problème similaire en astronomie. Nous avons nous aussi choisi cette méthode, en le modifiant pour en améliorer les performances, car comme dans toute méthode du gradient, il est possible de se heurter au problème de valeurs négatives de $f_{n}$ après une itération.

Pour résoudre cette difficulté nous avons utilisé la procédure suivante : durant chaque itération, chaque fois qu'un élément de $f_{n}$ devient négatif, on le remet à une valeur positive très petite, et on annule la composante correspondante de son gradient. Ainsi on dévie le gradient en faveur des grandes valeurs de $f_{n}$.

Avec cette procédure nous avons aussi constaté une accélération dans la convergence de la méthode, d'autant plus sensible que l'objet à restaurer est constitué de plusieurs objets ponctuels.

3.5 Mise en ceuvre De L'Algorithme. - Nous avons appliqué cette méthode au problème de la reconstruction d'images à partir des mesures du champ diffracté, dans les deux situations suivantes :

3.5.1 Les $m_{i}$ sont directement les données mesurées du champ diffracté. Dans ce cas les éléments de la matrice $A$ sont :

$$
A_{\text {in }}=\exp \left[-j k_{0}\left(x_{0}-x_{n}^{\prime}\right)\right] G\left(x_{0}-x_{n}^{\prime}, y_{0}-y_{n}^{\prime}\right)
$$

où $G(x, y)$ est la fonction de Green donnée par $G(x, y)=(j / 4) H_{0}^{(1)}\left[k_{0}\left(x^{2}+y^{2}\right)^{1 / 2}\right]$. La difficulté dans ce cas est qu'il n'y a pas de propriétés particulières de cette matrice qui permettent une simplification importante $[6,8]$.

3.5.2 Les $m_{i}$ sont les données obtenues par TF des mesures du champ diffracté (synthèse de Fourier).
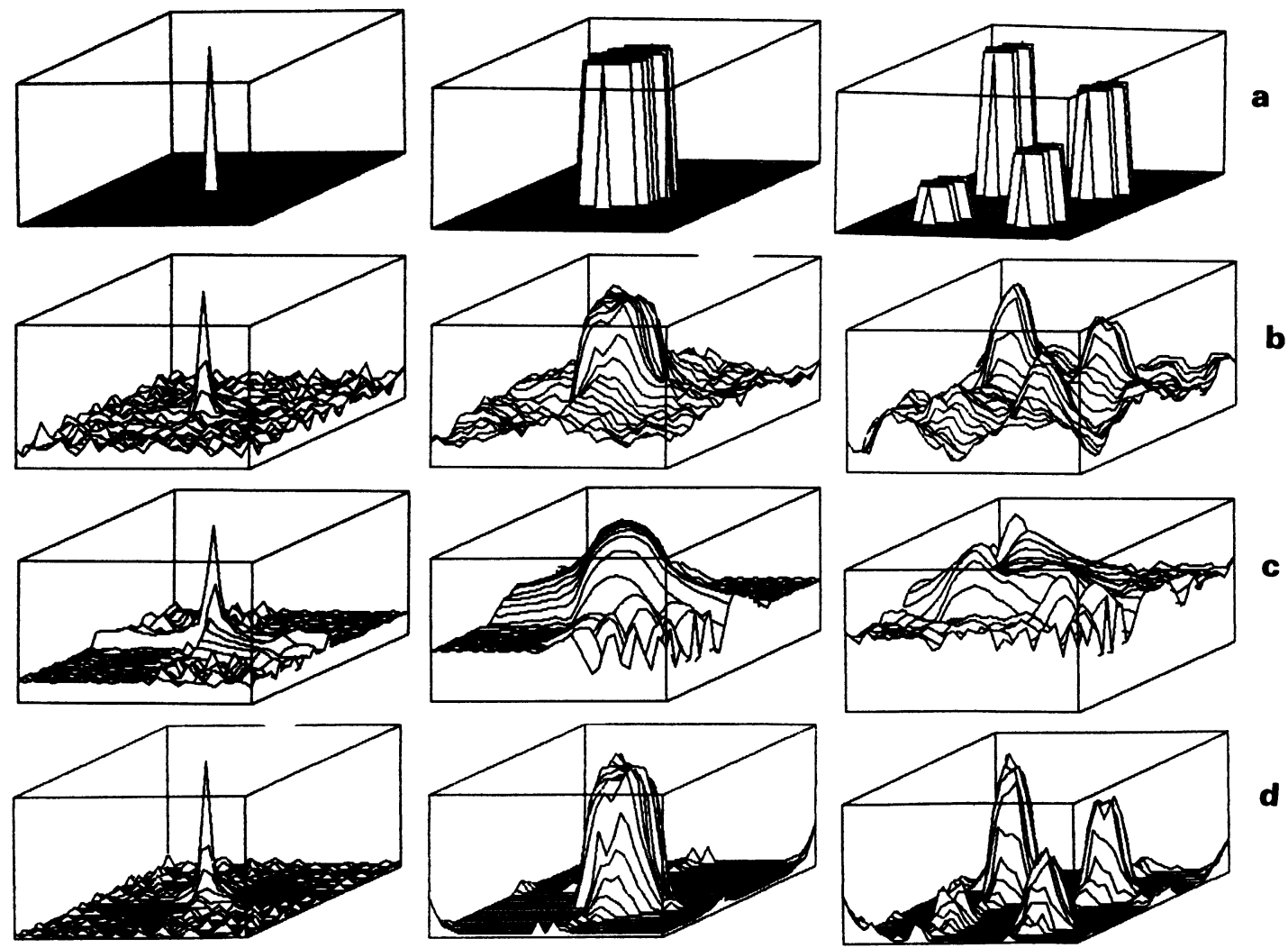

Fig. 6. - Comparaisons entre la méthode du ME et les méthodes classiques. a) objet à reconstruire, b) reconstruction par $\mathrm{M} 1, \mathrm{c})$ reconstruction par $\mathrm{M} 2$, et d) reconstruction par ME.

[Comparison between ME and classical methods. a) objects, b) M1 reconstructions, c) M2 reconstructions, and d) ME reconstructions.] 
Dans ce cas les éléments de la matrice $A$ sont :

$$
A_{i n}=\exp \left[-j\left(u_{i} x_{n}^{\prime}+v_{i} y_{n}^{\prime}\right)\right]
$$

Dans ce cas par contre, la matrice $A$ est une matrice de TF. On peut alors effectuer un certain nombre de simplifications dans le calcul de $Q(\mathbf{f})$ et de sa dérivée $\partial Q(\mathbf{f}) / \partial f_{n}$.

\section{L'algorithme général.}

L'algorithme général de reconstruction est le suivant :

1) estimation initiale $f_{n}^{(0)}=(1 / N) \sum m_{i} A_{i n^{*}}^{*}$ où $A_{i n}^{*}$ est le complexe conjugué de $A_{i n}$. Dans le cas de la synthèse de Fourier, $f_{n}^{(0)}$ est en fait la reconstruction directe par la TFD.

2) calcul de $J(\mathbf{f})=H(\mathbf{f})+\lambda Q(\mathbf{f})$ et $\nabla J(\mathbf{f})$. Dans cette étape il faut d'abord s'assurer de la positivité de tous les $f_{n}$ dans l'itération précédente. Pour ceci tous les $f_{n}$ négatifs sont remplacés par une valeur positive petite, et en même temps on remet à zéro les composantes correspondantes du gradient $\left(\partial J(f) / \partial f_{n}\right)$. D'autre part, dans le cas général, le calcul de $Q(f)$ et de son gradient s'effectue à l'aide des équations (17), tandis que dans le cas de la synthèse de Fourier le volume des calculs peut être réduit à l'aide des équations (18).

3) calcul de f par la méthode du gradient conjugué

4) calcul des critères d'arrêt ; par exemple le critère

$$
E=\sum\left|m_{i}-\hat{m}_{i}\right|^{2} / \sum\left|m_{i}\right|^{2},
$$

et d'autres tests de bon fonctionnement de l'algorithme à chaque itération. Ceci en plus des critères d'arrêt et des tests de bon fonctionnement de l'algorithme du gradient. Par exemple le critère de test suivant

$$
\mathrm{TEST}=(1 / 2)|(\nabla H /|\nabla H|)-(\nabla Q /|\nabla Q|)|
$$

nous permet de mesurer le degré de non-paralléllisme entre $\nabla H$ et $\nabla Q$ qui doit rester proche de zéro.

5) test d'arrêt ou de retour en 3 pour l'itération suivante.

3.6 Résultats de Simulations. - Dans ces simulations nous nous sommes limités au problème de la synthèse de Fourier. Pour chaque objet nous avons calculé sa TF sur des arcs de cercles pour simuler le

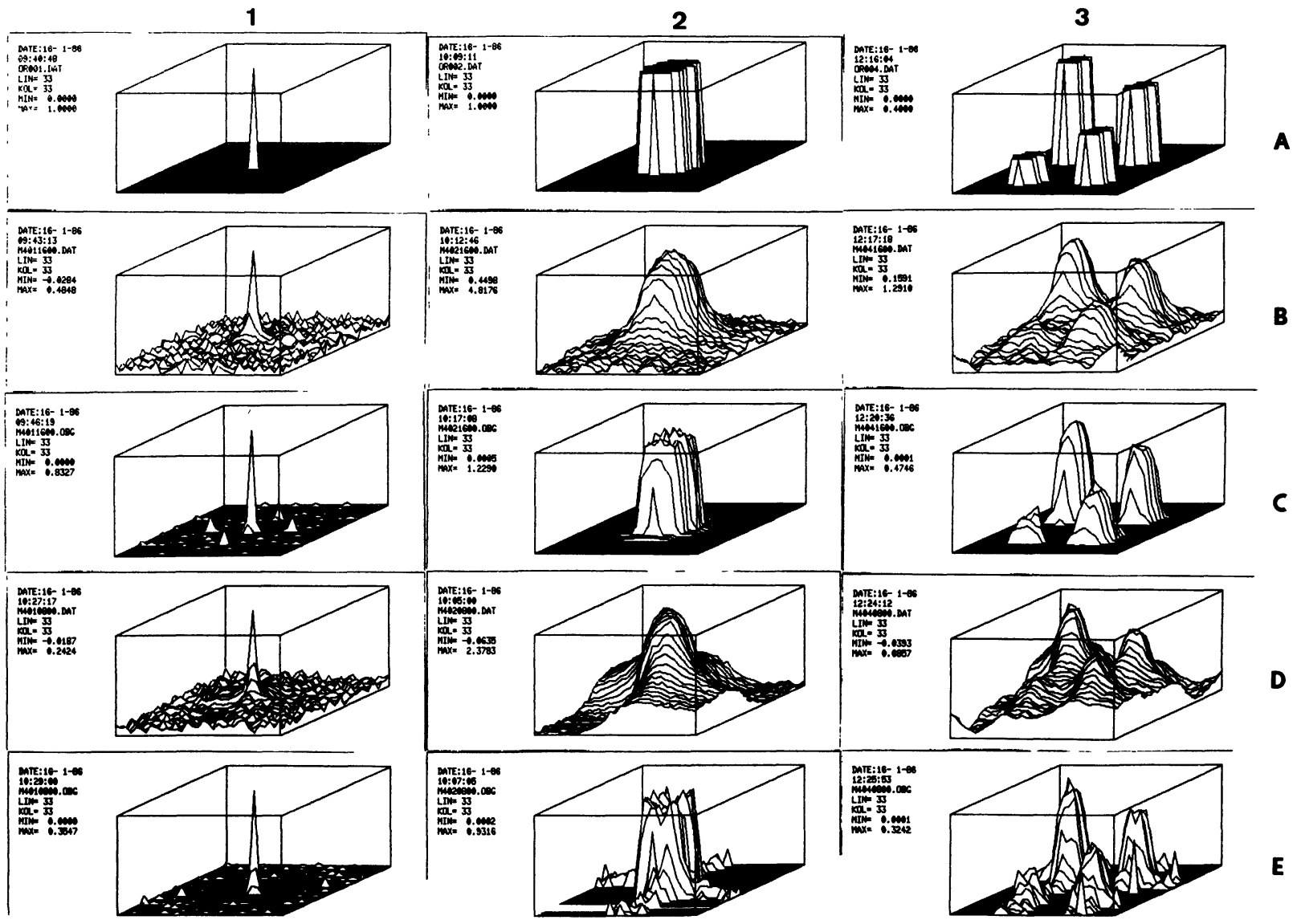

Fig. 7. - Reconstruction par M1 (b, d) et par ME (c, e), d'un objet réel (a). (b, c) $N_{\mathrm{P}}=8, \theta=360^{\circ}$, (d, e) $N_{\mathrm{P}}=8, \theta=90^{\circ}$.

[Reconstructions by $\mathrm{M} 1$ (b, d) and $\mathrm{ME}(\mathrm{c}, \mathrm{e})$ of a real object (a). (b, c) $N_{\mathrm{P}}=8, \theta=360^{\circ},(\mathrm{d}, \mathrm{e}) N_{\mathrm{P}}=8, \theta=$ $90^{\circ}$.] 


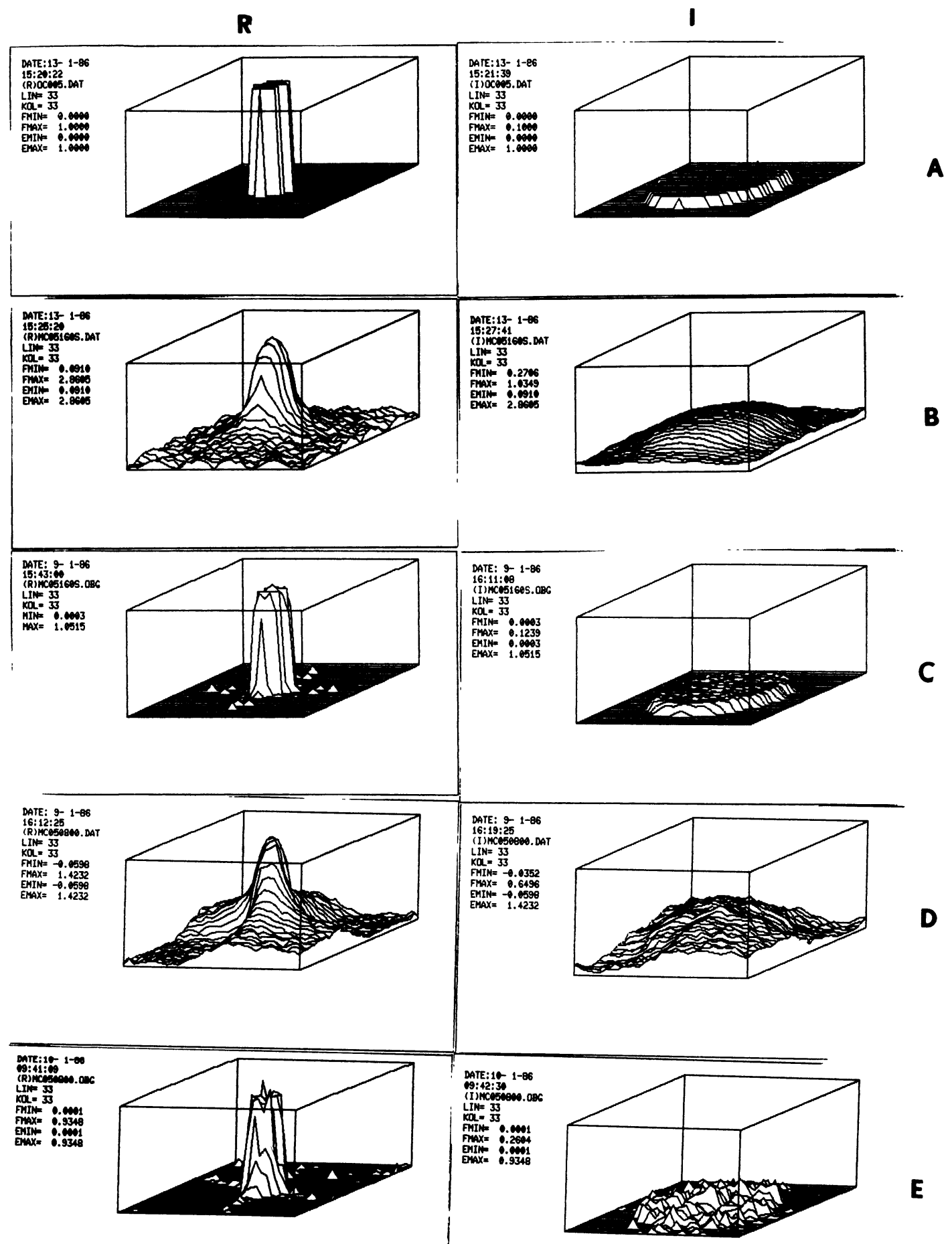

Fig. 8. - Reconstruction par M1 (b, d) et par ME (c, e), d'un objet complexe (a) dont la partie réelle (R) et la partie imaginaire (I) sont considérées comme positives et indépendantes. (b, c) $N_{\mathrm{P}}=8, \theta=360^{\circ}$, (d, e) $N_{\mathrm{P}}=8, \theta=90^{\circ}$.

[Reconstructions by M1 (b, d) and ME (c, e) of a complex object (a) in which the real (R) and imaginary (I) parts are considered as positive and independent quantities. $(\mathrm{b}, \mathrm{c}) N_{\mathrm{P}}=8, \theta=360^{\circ}$, (d, e) $N_{\mathrm{P}}=8, \theta=90^{\circ}$.]

problème de la tomographie à onde diffractée. Ceci nous permet de nous affranchir des autres difficultés, en particulier du problème de l'approximation linéaire des équations de propagation, ainsi que des difficultés de passage du domaine des mesures au domaine de Fourier. Ensuite, partant de ces données dans le domaine de Fourier, nous avons utilisé pour effectuer la reconstruction soit les méthodes classiques de l'interpolation dans le domaine de Fourier M1 ou dans le domaine spatial M2, soit notre méthode de ME.

Nous nous sommes placés dans un cas difficile où les vues sont non seulement limitées en nombre $\left(N_{\mathrm{P}}=8\right)$, mais de plus réparties dans un angle limité à $90^{\circ}$, et nous avons effectué deux séries de simulations : 
3.6.1 Comparaisons entre la méthode de $\mathrm{ME}$ et les méthodes classiques. - Les figures suivantes montrent ces résultats. Pour ces simulations nous avons supposé que l'objet est une grandeur réelle et positive (Fig. 6). Dans cette figure

a) est l'objet à reconstruire,

b) est la reconstruction par M1,

c) est la reconstruction par M2, et

d) est la reconstruction par ME.

3.6.2 Performances de la méthode de ME. - Dans ces simulations nous avons comparé les résultats de la reconstruction par $\mathrm{ME}$ aux résultats de la reconstruction par la méthode directe de TF inverse (Méthode M1 avec une interpolation d'ordre zéro) (Figs. 7 et 8).

Dans la figure 7 l'objet est une grandeur réelle et positive, et :

a) est l'objet à reconstruire,

b) est la reconstruction par TF inverse pour $N_{\mathrm{P}}=8$, $\theta=360^{\circ}$,

c) est la reconstruction par $\mathrm{ME}$ pour $N_{\mathrm{P}}=8$, $\theta=360^{\circ}$,

d) est la reconstruction par TF inverse pour $N_{\mathrm{P}}=8$, $\theta=90^{\circ}$

e) est la reconstruction par $\mathrm{ME}$ pour $N_{\mathrm{P}}=8$, $\theta=90^{\circ}$

Dans la figure 8 l'objet est une grandeur complexe et nous faisons l'hypothèse que ses parties réelle et imaginaire sont indépendantes. Dans cette figure :

a) représente la partie réelle et la partie imaginaire de l'objet à reconstruire,

b) est la reconstruction par TF inverse pour $N_{\mathrm{P}}=8$, $\theta=360^{\circ}$,

c) est la reconstruction par $\mathrm{ME}$ pour $N_{\mathrm{P}}=8$, $\theta=360^{\circ}$,

d) est la reconstruction par TF inverse pour $N_{\mathrm{P}}=8$, $\theta=90^{\circ}$,

e) est la reconstruction par ME pour $N_{\mathrm{P}}=8$, $\theta=90^{\circ}$,

Ces résultats de simulation montrent l'intérêt de notre méthode de reconstruction. Elle permet en effet d'obtenir à la fois un gain considérable de résolution en amplitude et de résolution spatiale dans les images reconstruites par rapport aux résultats obtenus par les méthodes classiques existantes.

Finalement, un exemple de simulation en imagerie micro-onde est donné à la figure 9. Il s'agit de mettre en évidence l'effet d'une variation de la température sur les caractéristiques électromagnétiques d'un bras contenant une tumeur. Ce fantôme a été élaboré par la division Ondes du Laboratoire des Signaux et Systèmes (LSS) qui développe un système d'imagerie médicale par tomographie micro-onde [6]. Dans cette figure

a) représente l'image d'un bras à $0^{\circ} \mathrm{C}$, l'image à $5{ }^{\circ} \mathrm{C}$ et leur différence qui permet de mieux distinguer la tumeur,

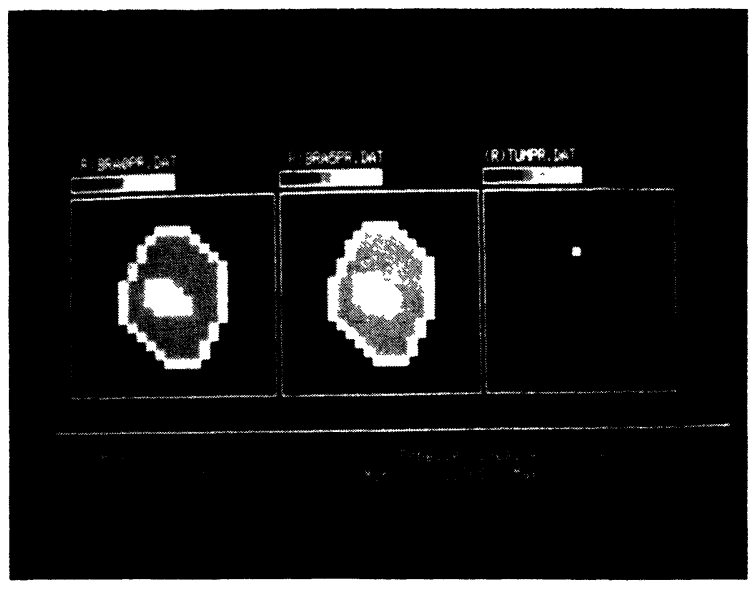

a)

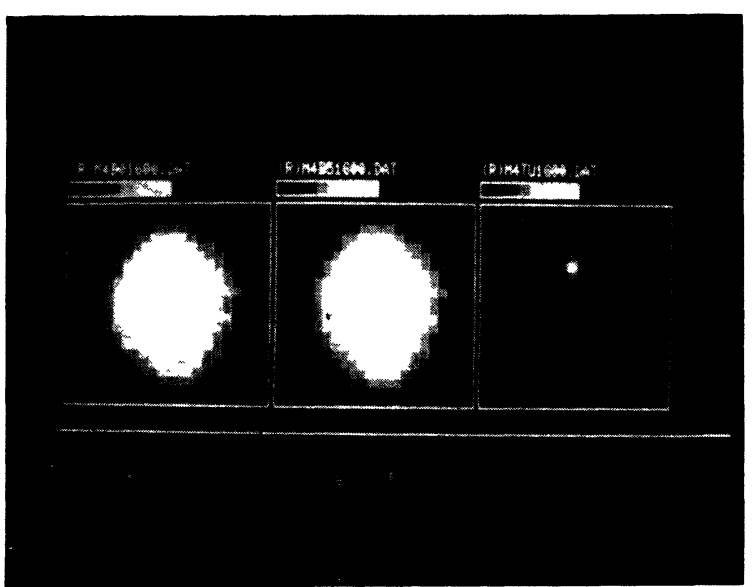

b)

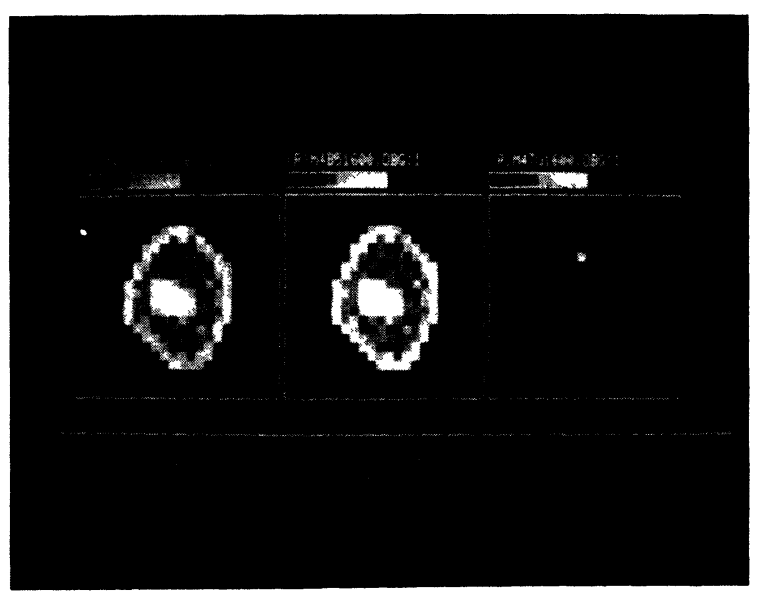

c)

Fig. 9. - Simulation d'un fantôme de bras en imagerie microondes; a) fantôme à $0^{\circ} \mathrm{C}$, à $5^{\circ} \mathrm{C}$, et leur différence mettant en évidence la tumeur ; b) reconstruction par M1 $\left(N_{\mathrm{P}}=16\right.$, $\left.\left.\theta=360^{\circ}\right) ; c\right)$ reconstruction par $\operatorname{ME}\left(N_{\mathrm{P}}=16, \theta=360^{\circ}\right)$.

[A fantom simulation of an arm in microwave diffraction tomography, a) fantoms at $0{ }^{\circ} \mathrm{C}, 5^{\circ} \mathrm{C}$, and their difference improving the visibility of a tumor ; b) M1 reconstructions $\left(N_{\mathrm{P}}=16, \theta=360^{\circ}\right) ;$ c) $\mathrm{ME}$ reconstructions $\left(N_{\mathrm{P}}=16\right.$, $\left.\theta=360^{\circ}\right)$.] 
b) représente les trois images reconstituées par TF inverse pour $N_{\mathrm{P}}=16, \theta=360^{\circ}$.

c) représente les trois images reconstituées par la méthode du ME pour $N_{\mathrm{P}}=16, \theta=360^{\circ}$.

On constate l'amélioration de la résolution apportée par notre méthode.

\section{Conclusions.}

Les méthodes classiques de reconstruction d'images à partir de leur connaissance partielle dans le domaine de Fourier (synthèse de Fourier) par des techniques d'interpolation (dans le domaine de Fourier ou dans le domaine spatial) ont deux grands inconvénients à la fois théorique et pratique. Nous avons proposé une méthode de reconstruction basée sur le principe du ME dans laquelle

- toutes les prédictions de la méthode sont consistantes avec les données mesurées et les informations $a$ priori que l'on a sur la solution;

- l'adoption d'une contrainte statistique pour prendre en compte l'incertitude sur les données plutôt que des contraintes individuelles sur ces données garantit l'existence d'une solution qui est consistante avec ces données dans la limite de leurs incertitudes.

\section{Bibliographie}

[1] Wolf, E., Three-dimensional structure determination of semi-transparent objects from holographic data, Opt. Commun. 1 (1969) 153-156.

[2] DevanEY, A. J., A filtered backpropagation algorithm for diffraction tomography. Ultrason. Imag. 4, (1982) 336-350.

[3] BOJARSKI, N. N., Inverse scattering inverse source theory. J. Math. Phys. 22 (1981) 1647-1650.

[4] Richmond, J. H., Scattering by a dielectric cylinder of arbitrary cross-section shape. IEEE Trans. Vol. AP-13 (1965) 334-341.

[5] Slaney, M., KaK, A. C., Larsen, L. E., Limitations of the Imaging with First-Order Diffraction Tomography. IEEE Trans. MIT-32 (1984) 860874.

[6] Pichot, Ch., Jofre, L., Peronnet, G., Bolomey, J., Active Microwave Imaging of Inhomogeneous bodies. IEEE Trans AP-33 (1985) 416-425.

[7] PAN, S. X., KAK, A. C., A Computational Study of Reconstruction Algorithms for Diffraction Tomography: Interpolation Versus Filtered Backpropagation. IEEE Trans ASSP-31 (1983) 1262-1275.

[8] Mohammad-Djafari, A., Demoment, G., Problèmes liés à la reconstruction d'images par tomographie micro-onde et ultrasonore. Proc. of 10th Coll. GRETSI, Nice, (1985) 701-706.

[9] Sanz, J. L., HuAng, Th. S., Discrete and Continuous Band-limited Signal Extrapolation IEEE Trans. ASSP-31, No. 5 (1983) 1276-1285.
[10] SANZ, J. L. C., On the Reconstruction of Bandlimited Multidimensional Signals from Algebraic Sampling Contours IBM Research Report RJ 4351 (47429), (1984).

[11] Johnson, R., \& SHORE, J., Which is Better Entropy Expression for Speech Processing : - S log S or log S ?, IEEE Trans. Vol. ASSP-32 (1984) 129137.

[12] Wernecke, S. J., D'Addario, L. R., Maximum Entropy Image Reconstruction. IEEE Trans C26 (1977) 351-364.

[13] Frieden, B., Statistical Models for the Image Restoration Problem, Comp. Graph. Imag. Proc. 12 (1980) 40-59.

[14] KIKUCHI, R., and SofFER, B. H., Maximum entropy image restoration $\mathrm{I}$. The entropy expression. $J$. Opt. Soc. Am. 67 (1977) 1656-1665.

[15] Daniell, G. J., Gull, S. F., Maximum Entropy algorithm applied to image enhancement, Proc. IEE 127E (1980) 170-172.

[16] Fletcher, R., Reeves, C. M., Function minimization by conjugate gradients, Comp. J. 7 (1964) 149-157.

[17] PollaK, E., Computational methods in optimization : a unified approach (Academic Press, London) 1971.

[18] Powell, M. J. D., Restart Procedure for the Conjugate Gradient method, Math. Prog. 12 (1977) 241-254. 